\title{
Doença vestibular central por infarto isquêmico secundário a hipotireoidismo em cão
}

\author{
Central Vestibular Disease Due to Ischemic Stroke Secondary to Hypothyroidism in a Dog \\ Rafael Oliveira Chaves', Marcelo Luis Schwab², Angel Ripplinger², Bruna Copat ${ }^{3}$, Graciane Aiello², \\ Denis Antonio Ferrarin ${ }^{2}$, Mariana Martins Flores ${ }^{4}$, Rafael Fighera ${ }^{4}$ \& Alexandre Mazzanti ${ }^{5}$
}

\begin{abstract}
Background: Hypothyroidism in dogs can cause lethargy, weight gain, dermatological signs and, uncommonly, neurological signs. These may involve the peripheral or central nervous system, leading to a decreased level of consciousness, central or peripheral vestibular disease, epileptic seizures, cognitive dysfunction, facial nerve paralysis, laryngeal paralysis, and polyneuropathy. There are few cases reported in the literature relating hypothyroidism and central vestibular disease. The aim of this study was to report a case of a stroke secondary to hypothyroidism that resulted in central vestibular syndrome in a dog. Case: A 13-year-old female Pitbull, weighting $37 \mathrm{~kg}$, was referred to a Veterinary University Hospital with a two weeks history of dyspnea, coughing and walking difficulty. On neurological examination, the animal presented vertical nystagmus, somnolence, non-ambulatory tetraparesis, decreased postural reaction in all limbs and normal spinal reflexes. The laboratory exams revealed a mild leukocytosis and increased creatinine, glucose, cholesterol and triglycerides levels. Due to historical, clinical, neurological and laboratory tests findings, the animal was diagnosed with central vestibular syndrome secondary to an intracranial neoplasm. Clinical treatment was instituted with corticosteroids, however the animal did not respond and died. On necropsy, the heart was enlarged and coronary arteries were thicker. No macroscopic changes were found in the brain. On histological analysis, the coronary arteries were congest by lipids that almost occluded the arterial lumen. The same alteration were found in splenic and renal arterioles, and in the brain's leptomeninges. Also in the brain, it was observed perivascular infiltration of mononuclear cells and focal gliosis. The thyroid was atrophied and fibrosed bilaterally. Therefore, those changes were compatible with atherosclerosis secondary to hypothyroidism.

Discussion: Neurological sings secondary to hypothyroidism are uncommon. From 116 dogs with hypothyroidism, only 22 (19\%) had neurological presentations. The majority was associated with peripheral nervous system showing polyneuropathy and cranial nerve dysfunction. When associated with central nervous system the most common sings are pathological nystagmus, postural reaction deficits, tetraparesis, hemiparesis, and paradoxical vestibular diseases. These signs are similar to the findings in the case reported here, characterizing it as a central vestibular disease. Different from humans, dogs have a natural resistance against atherosclerosis, however, hypothyroidism can result in lipids metabolic deficits, increasing its plasma levels. In this report, the arterial lesion and ischemia secondary to atherosclerosis, especially in the brain steam, have led to the neurological presentation. The dog also had hypercholesterolemia and hypertriglyceridemia, which are related to hypothyroidism. As high levels of cholesterol and triglycerides are common findings in hypothyroidism, it is important to evaluating blood levels of those lipids in dogs with central vestibular diseases, even though there are no others endocrinal signs. This case shows that, even though central vestibular disease is not a common neurological presentation in hypothyroidism, it is always important to considerer this metabolic affection as a precursor and a differential diagnosis to central vestibular disease.
\end{abstract}

Keywords: atherosclerosis, stroke, vestibular disease, canine.

Descritores: aterosclerose, infarto isquêmico, doença vestibular, cães. 


\section{INTRODUÇÃO}

O sistema vestibular é o principal componente do sistema nervoso responsável pela manutenção do equilíbrio. Além disso, coordena os movimentos da cabeça (incluindo movimentos oculares), do tronco e dos membros [11]. As porções periféricas do sistema vestibular estão localizadas na orelha interna e consistem de receptores ciliados de neurônios sensoriais bipolares e axônios periféricos da divisão vestibular do VIII nervo craniano (nervo vestibulococlear). As centrais estão localizadas nos núcleos vestibulares da medula oblonga e nas projeções vestibulares para o cerebelo, medula espinhal e medula oblonga rostral [11].

Alterações neurológicas secundárias ao hipotireoidismo são incomuns em cães, principalmente quando comparadas a outros sinais clínicos como letargia, ganho de peso e alterações dermatológicas [4,7]. Quando presentes, podem envolver o sistema nervoso periférico ou central e os mais comuns incluem: diminuição do nível de consciência, andar em círculos, doença vestibular, crises epilépticas, disfunção cognitiva, paralisia de nervo facial, paralisia de laringe e polineuropatia [1,2,4,6,8,13].

Em um estudo sobre doença vestibular em 81 casos, não foi encontrado nenhum caso de hipotireoidismo ocasionando doença vestibular central [3]. Além disso, mesmo na literatura consultada são poucos os casos demonstrados $[2,4,7,13,14]$. Com o relato do caso a ser descrito, pretende-se demonstrar que o acidente vascular encefálico (infarto isquêmico), embora infrequente, pode ocasionar síndrome vestibular central e ter, como causa indireta, o hipotireoidismo.

\section{CASO}

Foi atendida no Hospital Veterinário Universitário de uma Instituição, uma cadela Pit bull, com 13 anos de idade, pesando $37 \mathrm{~kg}$, com histórico de dispnéia mista, tosse e dificuldade para caminhar há duas semanas, com piora nos últimos três dias. No exame neurológico foi observado nistagmo vertical, sonolência, tetraparesia não ambulatória, reações posturais (propriocepção e teste do salto) diminuídas nos quatro membros, reflexos segmentares espinhais em membros pélvicos (patelar, flexor e perineal) e toráci$\cos$ (flexor) e tônus muscular normais, caracterizando doença vestibular central.

Os exames complementares realizados incluíram: hemograma (Ht 48,6\% [37-55]; hemoglobina
16 g dL-1 [12-18]; VCM 73,2 fL [60-77], CHCM 34,1\% [32-36]; leucócitos totais $17.200 \mu \mathrm{L}-1$ [600017000]; plaquetas: $404.000 \mu \mathrm{L}-1$ [200.000-500.000]; bioquímica sérica (Creatinina: 1,9 mg dL-1 [0,5-1,5]; Uréia: 60,12 mg dL-1 [21-59]; ALT: 95 UI L-1 [21102]; AST: 58 UI L-1 [23-66]; FA: 148 UI L-1 [20156]; Glicose: 122 mg dL-1 [70-110]; Proteínas totais: 6,8 g dL-1 [5,4-7,1]; Albumina: 3,03 g dL-1 [2,6-3,3]; Colesterol: 861 mg dL-1 [125-300]; Triglicerídeos: 554 mg dL-1 [15-380]); urinálise sem alterações; ultrassom de abdome (hepatomegalia discreta) e radiografia simples do tórax (cardiomegalia generalizada com deslocamento dorsal da traqueia). Os níveis dos hormônios T4 total, T4 Livre e TSH foram 0,74 mcg/dL (1,4-3,8), $0,38 \mathrm{ng} / \mathrm{dL}(0,50-1,60)$ e $0,52 \mathrm{ng} / \mathrm{mL}(0,04-0,40)$, respectivamente, compatíveis com hipotireoidismo [10].

Como principais diagnósticos diferenciais foram listados: neoplasma, doença inflamatória infecciosa, não infecciosa e vascular. Foi instituído o tratamento clínico com corticosteroide, porém o animal apresentou piora do quadro clínico e foi a óbito. $\mathrm{Na}$ necropsia, foi observado aumento moderado do coração e artérias coronárias esbranquiçadas, espessadas e com parede externa irregular (Figura 1A). Na parede da aorta, observou-se um nódulo exofítico de aproximadamente três centímetros de diâmetro. Havia múltiplas áreas escuras, levemente elevadas, no baço. Ao corte, eram macias, bem circunscritas e vermelhoescuras (infartos esplênicos). Não foram observadas alterações macroscópicas no encéfalo.

No estudo histológico do coração, observou-se uma acentuada desorganização na parede das artérias coronárias que estavam acentuadamente espessadas, quase ocluindo o lúmen arterial. As áreas de desorganização e espessamento da parede eram caracterizadas por acúmulo de lipídeos (no interior de macrófagos espumosos, no interior de células musculares da camada média ou livre no interstício, visto na forma de gotículas ou fendas de colesterol), e focos de mineralização. Em um dos vasos, foi encontrado um trombo em organização, caracterizado por proliferação de tecido fibroso, recanalização e presença de macrófagos em meio ao trombo (Figura 1B). A mesma alteração observada nas artérias coronárias foi encontrada em grande quantidade de arteríolas esplênicas (Figuras 1C e D), renais e nas leptomeninges do encéfalo, principalmente na região do tronco encefálico (Figura 1E). 


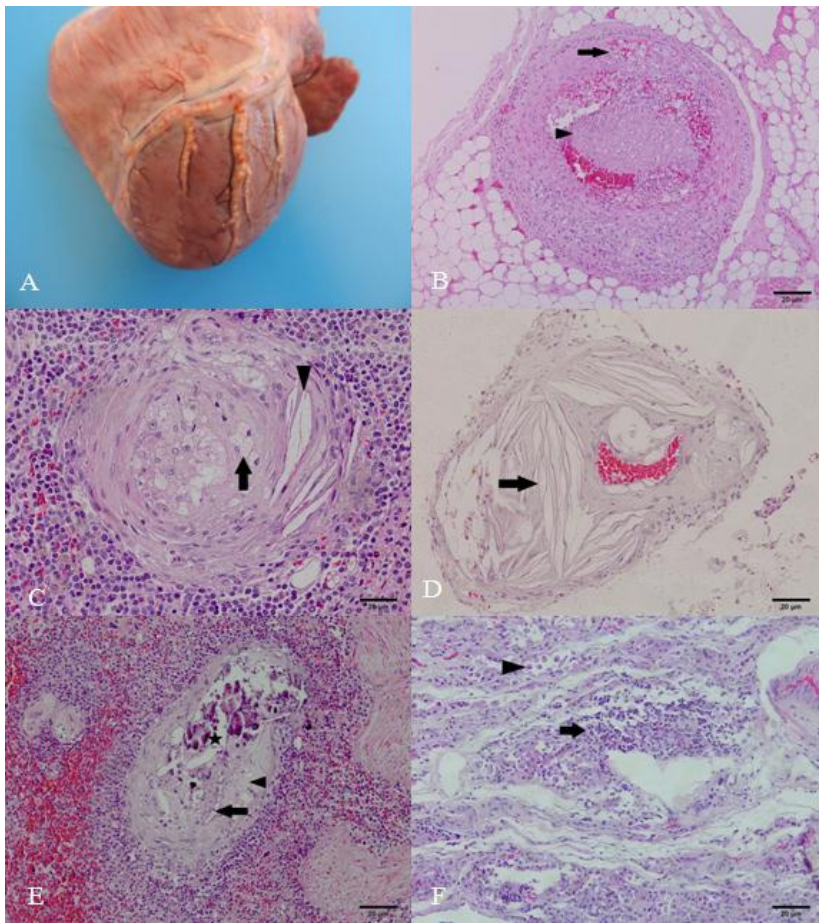

Figura 1. A- Imagem fotográfica do coração de um cão com doença vestibular central por infarto isquêmico secundário a hipotireoidismo. Notam-se as artérias coronárias esbranquiçadas, acentuadamente espessadas e com superfície externa irregular. B- Artéria coronária. Espessamento acentuado da parede caracterizado principalmente pelo acúmulo de macrófagos espumosos repletos de gotículas de lipídios (seta). Observa-se um trombo aderido à superfície endotelial (cabeça da seta), ocluindo o lúmen vascular [HE Obj. 20x]. C- Arteríola esplênica. Presença de macrófagos espumosos (seta) e fendas de colesterol (cabeça da seta) causando espessamento da parede e oclusão do lúmen [HE Obj. 40x]. D- Artéria das leptomeninges. Espessamento da parede com áreas dissecadas predominantemente pela formação de fendas de colesterol (seta) [HE Obj. 40x]. E- Arteríola esplênica. Lúmen vascular ocluído (seta) por acúmulo de macrófagos espumosos (cabeça da seta) e formação fendas de colesterol (estrela) focalmente mineralizadas [HE Obj. 20x]. F- Tireoide. Presença de acentuado infiltrado inflamatório linfocítico (seta) com raras células do epitélio folicular remanescentes (cabeça da seta), caracterizando uma tireoidite linfocitária [HE Obj. 10x].

No encéfalo, observou-se também, áreas de infiltrado inflamatório mononuclear perivascular e áreas de gliose focal, além de vacuolização da substância branca de várias secções, mas particularmente marcada no cerebelo. As alterações observadas nas tireoides foram interpretadas como uma tireoidite linfocítica difusa acentuada, com atrofia severa e fibrose bilateral (Figura 1F). Logo, as alterações microscópicas observadas em múltiplas artérias desse cão foram compatíveis com aterosclerose secundária ao hipotireoidismo primário.

\section{DISCUSSÃO}

A prevalência de hipotireoidismo em cães adultos e idosos foi estimada entre $0,2 \%$ e $0,8 \%[4,9,10]$ e a média de idade para cães com sinais vestibulares centrais devido ao hipotireoidismo foi de sete anos
[7]. Embora alguns autores não relatam predisposição racial [4], cães de raças grandes parecem ser mais predispostos [1]. O cão do presente relato era de grande porte, porém com idade superior a encontrada na literatura como mais prevalente.

A análise de 116 cães com hipotireoidismo, sinais neurológicos foram relatados em 22 (19\%) casos, sendo alterações no sistema nervoso periférico, como polineuropatia e disfunção de nervos cranianos, as mais comuns [4]. Assim como no estudo de Higgins et al. [7], o cão desse relato apresentava sinais de doença vestibular central (diminuição do nível de consciência, deficiência nas reações posturais e nistagmo vertical), os quais resultaram provavelmente do infarto isquêmico no tronco encefálico. Segundo esse mesmo autor, os sinais neurológicos mais comuns incluíram: nistagmo patológico, deficiência na reação postural, tetraparesia, hemiparesia e doença vestibular paradoxal (caracterizada por inclinação de cabeça contralateral as deficiências nas reações posturais) e a progressão da doença foi variável, sendo que $80 \%$ apresentaram início agudo dos sinais neurológicos. No paciente em questão, assim como citado por Higgins et al. [7], a apresentação dos sinais neurológicos foi aguda, no entanto, observou-se evolução crônica e progressiva dos sinais sistêmicos. Segundo o proprietário, o animal estava mais apático e letárgico nos últimos 12 meses, características que estão normalmente presentes no hipotireoidismo, devido à diminuição da taxa metabólica (15\% em comparação com cães normais) [9], e também pelo acúmulo de mucopolissacarídeos no tecido subcutâneo (mixedema), apontado como responsável pelo ganho de peso nesses animais [1].

Em humanos, o aumento nas concentrações de lipídeos de baixa densidade foi relacionado com maior risco de desenvolvimento de aterosclerose [6]. Em cães, mesmo havendo uma resistência natural ao desenvolvimento de aterosclerose [5], o hipotireoidismo pode ocasionar deficiência no metabolismo lipídico, resultando em acúmulo no plasma [6]. Cães com hipotireoidismo foram $51 \%$ mais propensos a desenvolver aterosclerose [6]. No cão desse relato, a lesão arterial e consequente isquemia observada no encéfalo, particularmente no tronco encefálico (medula oblonga rostral), secundárias a aterosclerose, resultaram nos sinais neurológicos. Adicionalmente, a elevação nas concentrações de triglicerídeos e colesterol, como observadas nesse caso, contribuíram para o 
aumento da viscosidade do sangue e o risco de eventos tromboembólicos [6,12,13].

Hipercolesterolemia e hipertrigliceridemia ocorre em $75 \%$ e $88 \%$, respectivamente, dos cães com hipotireoidismo, devido ao aumento das concentrações de lipoproteínas de baixa densidade [9]. Além disso, hiperglicemia também podem ser observadas em $49 \%$ dos cães com hipotireoidismo [9] e em muitos casos pode estar relacionada ao diabetes mellitus levando a aterosclerose [6]. O cão do presente relato apresentava hipercolesterolemia, hipertrigliceridemia e hiperglicemia. Assim, devido à alta correlação entre colesterol e triglicerídeos com o hipotireoidismo, os autores desse trabalho recomendam investigar os níveis séricos desses lipídios em cães adultos e idosos com alterações vestibulares centrais, mesmo na ausência de outros sinais que fariam suspeitar dessa endocrinopatia.

Diante do exposto acima, o caso traz como relevância clínica, demonstrar as alterações encontradas em um cão com sinais vestibulares centrais e evidenciar o hipotireoidismo como causa indireta de doença vestibular central, mesmo que essa afecção metabólica raramente acometa o sistema nervoso central.

Declaration of interest. The authors report no conflicts of interest. The authors alone are responsible for the content and writing of the paper.

\section{REFERENCES}

1 Bertalan A., Kent M. \& Glass E. 2013. Neurologic manifestations of hypothyroidism in dogs. Compendium on continuing education for the practicing veterinarian. 35(3): E1-E7.

2 Bichsel P., Jacobs G. \& Oliver Jr. J.E. 1988. Neurologic manifestations associated with hypothyroidism in 4 dogs. Journal of the American Veterinary Medical Association. 192(12): 1745-1747.

3 Chaves R.O., Beckmann D.V., Copat B., Souza F.W., Fabretti A.K., Gomes L.A., Fighera R.A. \& Mazzanti A. 2014. Doença vestibular em cães: 81 casos (2006-2013). Pesquisa Veterinária Brasileira. 34(12): 1231-1235.

4 Dixon R.M., Reid S.W. \& Mooney C.T. 1999. Epidemiological, clinical, haematological and biochemical characteristics of canine hypothyroidism. The Veterinary Record. 145(17): 481-487.

5 Downs L.G, Bolton C.H., Crispin S.M. \& Wills J.M. 1993. Plasma lipoprotein lipids in five different breeds of dogs. Research in Veterinary Science. 54(1): 63-67.

6 Hess R.S., Kass P.H. \& Van Winkle T.J. 2003. Association between diabetes mellitus, hypothyroidism or hyperadrenocorticism, and atherosclerosis in dogs. Journal of Veterinary Internal Medicine. 17(4): 489-494.

7 Higgins M.A., Rossmeisl Jr. J.H. \& Panciera D.L. 2006. Hypothyroid-associated central vestibular disease in 10 dogs: 1999-2005. Journal of Veterinary Internal Medicine. 20(6): 1363-1369.

8 Jaggy A., Oliver J.E, Ferguson D.C., Mahaffey E.A. \& Glaus Jr. T. 1994. Neurological manifestations of hypothyroidism: A retrospective study of 29 dogs. Journal of Veterinary Internal Medicine. 8(5): 328-336.

9 Mooney C.T. 2011. Canine hypothyroidism: a review of aetiology and diagnosis. New Zealand Veterinary Journal. 59(3): 105-114.

10 Mooney C.T. 2017. Canine hypothyroidism. In: Etinger S.J., Feldman E.C. \& Côte E. (Eds). Textbook of Veterinary Internal Medicine. 8th edn. St. Louis: Elsevier, pp.4198-4227.

11 Sanders G.S. 2016. Disorders of hearing and balance: the vestibulocochlear nerve (CN VIII) and associated structures. In: Dewey C.W. \& Da Costa R.C. (Eds). Practical Guide to Canine and Feline Neurology. 3rd edn. New Delhi: Wiley Blackwell, pp.277-297.

12 Velcheva L., Antonova N., Dimitrov N. \& Ivanov I. 2006. Plasma lipids and blood viscosity in patients with cerebrovascular disease. Clinical Hemorheology and Microcirculation. 35(1-2): 155-157.

13 Vitale C.L. \& Olby N.J. 2007. Neurologic dysfunction in hypothyroid, hyperlipedemic Labrador retrievers. Journal of Veterinary Internal Medicine. 21(6): 1316-1322.

14Zeiss C.J. \& Waddle G. 1995. Hpothyroidism and atherosclerosis in dogs. Compendium on continuing education for the practicing veterinarian. 17(9): 1117-1128. 